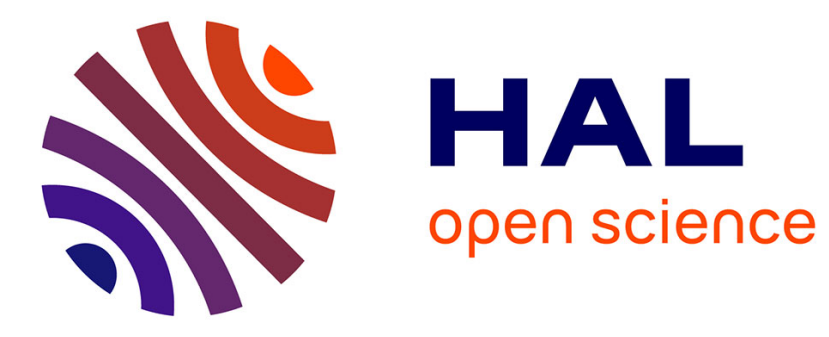

\title{
Synchronization in networks of linear singularly perturbed systems
}

Jihene Ben Rejeb, Irinel-Constantin Morarescu, Jamal Daafouz

\section{To cite this version:}

Jihene Ben Rejeb, Irinel-Constantin Morarescu, Jamal Daafouz. Synchronization in networks of linear singularly perturbed systems. American Control Conference, ACC'2016, Jul 2016, Boston, United States. hal-01294002

\section{HAL Id: hal-01294002 \\ https://hal.science/hal-01294002}

Submitted on 29 Mar 2016

HAL is a multi-disciplinary open access archive for the deposit and dissemination of scientific research documents, whether they are published or not. The documents may come from teaching and research institutions in France or abroad, or from public or private research centers.
L'archive ouverte pluridisciplinaire HAL, est destinée au dépôt et à la diffusion de documents scientifiques de niveau recherche, publiés ou non, émanant des établissements d'enseignement et de recherche français ou étrangers, des laboratoires publics ou privés. 


\title{
Synchronization in networks of linear singularly perturbed systems
}

\author{
Jihene Ben Rejeb, Irinel-Constantin Morărescu and Jamal Daafouz
}

\begin{abstract}
This work is motivated by the fact that many real systems are characterized by two features. The first one is that they are obtained by interconnecting a bunch of simpler subsystems that have to synchronize in order to reach a global goal. The second one is that each subsystem presents dynamics that evolves on different time-scales. Taking into account the two features leads to the problem of synchronization in networks of singularly perturbed systems. In this work we are providing a preliminary study that considers the problem where each subsystem is linear and the network topology is represented by a connected undirected graph that is fixed in time. We show that we can proceed to a time-scale separation of the overall network dynamics and design the controls that synchronize the slow dynamics and the fast ones. Applying the joint control actions to the network of singularly perturbed systems we obtain an approximation of the synchronization behavior imposed for each scale. The methodology requires a variable transformation to overcome the fact that we are dealing with non-standard singularly perturbed systems. One example illustrates the synchronization behavior of linear singularly perturbed systems.
\end{abstract}

Index Terms-Multiagent systems; consensus; singularly perturbed systems.

\section{INTRODUCTION}

Physical systems are often characterized by several dynamical processes evolving on different time scales and influencing each other [1]. When several orders of magnitude differentiate the various time scale the analysis of the overall systems becomes more difficult. In this case, standard control techniques lead to ill-conditioned problems. To overcome this, singular perturbation theory [2], [3] propose to approximate the dynamics by decoupling the slow dynamical process of the faster ones. Consequently, the control design is also decoupled with respect to each time scale and then is proven that the joint actions performs well when applied to the overall system.

The analysis and control design for multiple time-scales systems attracted a lot of interest due to their various applications going from biological systems such as gene expression systems [4], neurons behavior [5] to engineering problems [6]. General stability and stabilization of linear and nonlinear singularly perturbed systems can be found in [2], [3]. For linear singularly perturbed systems a linear quadratic optimal control design is proposed in [7]. Stabilization and

This work was funded by the ANR project COMPACS - "Computation Aware Control Systems", ANR-13-BS03-004.

J. Ben Rejeb, I.-C. Morărescu, J.Daafouz are with Université de Lorraine, CRAN, UMR 7039 and CNRS, CRAN, UMR 7039, 2 Avenue de la Forêt de Haye, Vandœuvre-lès-Nancy, France. e-mails: jihene.ben-rejeb@univ-lorraine.fr, constantin.morarescu@univ-lorraine.fr, jamal.daafouzeuniv-lorraine.fr exponential stability of singularly perturbed linear switched systems is considered in [8], [9]. Various biological singularly perturbed systems are analyzed from a geometric perspective in [10].

The particularity of existing studies presented above is that they consider singularly perturbed systems as being stand alone systems. Motivated by the fact that biological as technological systems are often composed of several coupled singularly perturbed systems, we are considering, in this work, the problem of synchronization of singularly perturbed systems. When only one time-scale characterizes the systems dynamics, the problem of synchronization has been extensively studied [11], [12], [13], [14], [15]. However, the synchronization of singularly perturbed systems has not been treated. We point out that time-scale separation has been observed and analyzed in power networks [16], [17], [18] but the systems composing the grid evolve on only one time scale.

In this work we consider a network of singularly perturbed linear systems and we provide the methodology allowing at extending the stabilization control design to the synchronization one. Precisely, we show that we can proceed to a timescale separation of the overall network dynamics and design the controls that synchronize the slow dynamics and the fast ones. Next, applying the joint control actions to the network of singularly perturbed systems we obtain an approximation of the synchronization behavior imposed for each scale. The main difficulty that we have to overcome is that the matrix defining the fast dynamics in closed loop is not invertible.

In this preliminary study we consider that the coupling topology, between the systems composing the network, is fixed and undirected. Therefore, the control feedback necessary for synchronization will use only local information provided by this fixed coupling topology. Extensions to directed and time-varying topologies as well as generalization to nonlinear dynamics will be considered in our future works.

The rest of the paper is organized as follows. In Section II we introduce the basic concepts that are used throughout the paper and we formulate the problem under consideration. The main results, concerning the design of the controllers that synchronize the subsystems using only local information, are presented in Section III. One numerical example illustrating the results is provided in Section IV. The paper ends with some concluding remarks and perspectives.

\section{Notation}

The following standard symbols are used throughout the paper. $\mathbb{R}$ is the set of real numbers, $|\mathcal{A}|$ is the cardinality of a given finite set $\mathcal{A},\|x\|$ is the Euclidean norm of the vector 
$x$ and $\otimes$ denotes the Kronecker product of two matrices. We also denote by $I_{n} \in \mathbb{R}^{n \times n}$ the identity matrix of size $n$ and by $\mathbf{1}_{n}, \mathbf{0}_{n} \in \mathbb{R}^{n}$ the column vector whose components are all 1 and 0 , respectively. We also denote by $\mathbf{0}_{n \times m} \in \mathbb{R}^{n \times m}$ the matrix whose all components are 0 . The transpose of a given matrix $A$ is denoted by $A^{\top}$. We denote $\operatorname{diag}\left(A_{1}, \ldots, A_{n}\right)$ the block diagonal matrix having the matrices $A_{1}$ to $A_{n}$ on the diagonal and 0 everywhere else.

\section{PRELIMINARIES AND PROBLEM FORMULATION}

We consider a network of $n$ identical singularly perturbed linear systems. For any $i=1, \ldots, n$, the $i^{\text {th }}$ system at time $t$ is characterized by the state $\left(x_{i}(t), z_{i}(t)\right) \in \mathbb{R}^{n_{x}+n_{z}}$ and there exists a small $\epsilon>0$ such that its dynamics is given by:

$$
\left\{\begin{aligned}
\dot{x}_{i}(t) & =A_{11} x_{i}(t)+A_{12} z_{i}(t)+B_{1} u_{i}(t) \\
\epsilon \dot{z}_{i}(t) & =A_{21} x_{i}(t)+A_{22} z_{i}(t)+B_{2} u_{i}(t), \quad i=1, \ldots, n
\end{aligned}\right.
$$

where $u_{i} \in \mathbb{R}^{m}$ is the control input and

$$
\begin{array}{lll}
A_{11} \in \mathbb{R}^{n_{x} \times n_{x}}, & A_{12} \in \mathbb{R}^{n_{x} \times n_{z}} & B_{1} \in \mathbb{R}^{n_{x} \times m}, \\
A_{21} \in \mathbb{R}^{n_{z} \times n_{x}} & A_{22} \in \mathbb{R}^{n_{z} \times n_{z}}, & B_{2} \in \mathbb{R}^{n_{z} \times m}
\end{array}
$$

such that $\operatorname{rank}\left(B_{1}\right)=\operatorname{rank}\left(B_{2}\right)=m$.

A standard assumption, in singular perturbation theory, which aims at ensuring the well posedness of (1) is the following.

\section{Assumption 1: The matrix $A_{22}$ is invertible.}

With the network of $n$ systems we associate a graph $\mathcal{G}$ which is a couple $(\mathcal{V}, \mathcal{E})$ where $\mathcal{V}=\{1, \ldots, n\}$ represents the vertex set and $\mathcal{E} \subset \mathcal{V} \times \mathcal{V}$ is the edge set. In the sequel we suppose that the graph is undirected meaning that $(i, j) \in$ $\mathcal{E} \Leftrightarrow(j, i) \in \mathcal{E}$. We also assume that $\mathcal{G}$ has no self-loop (i.e. $\forall i=1, \ldots, n$ one has $(i, i) \notin \mathcal{E})$. A weighted adjacency matrix associated with $\mathcal{G}$ is $G=\left[g_{i, j}\right] \in \mathbb{R}^{n \times n}$ such that

$$
\left\{\begin{array}{l}
g_{i j}>0 \text { if }(i, j) \in \mathcal{E} \\
g_{i j}=0 \text { otherwise }
\end{array} .\right.
$$

The corresponding Laplacian matrix is $L=\left[l_{i j}\right] \in \mathbb{R}^{n \times n}$ defined by

$$
\left\{\begin{array}{l}
l_{i i}=\sum_{j=1}^{n} g_{i, j}, \forall i=1, \ldots, n \\
l_{i j}=-g_{i, j} \text { if } i \neq j
\end{array} .\right.
$$

By definition $L$ is symmetric and all of its rows sum are zero.

Definition 1: A path of length $p$ in the graph $\mathcal{G}=(\mathcal{V}, \mathcal{E})$ is a union of edges $\bigcup_{k=1}^{p}\left(i_{k}, j_{k}\right)$ such that $i_{k+1}=j_{k}, \forall k \in\{1, \ldots, p-1\}$. The node $j$ is connected with node $i$ in $\mathcal{G}=(\mathcal{V}, \mathcal{E})$ if there exists at least a path in $\mathcal{G}$ from $i$ to $j$ (i.e. $i_{1}=i$ and $j_{p}=j$ ). A connected graph is such that any of its two distinct elements are connected.

Assumption 2: The undirected graph $\mathcal{G}$ is connected.

Remark 1 (Basic properties of the Laplacian matrix [19]): Let $\lambda_{1} \leq \lambda_{2} \leq \ldots \leq \lambda_{n}$ be the eigenvalues of $L$. Then

- $\lambda_{1}=0$ is a simple eigenvalue of $L$ associated with the eigenvector $\mathbf{1}_{n}$.

- $\lambda_{2}>0$ as far as $\mathcal{G}$ is connected. In other words, $L$ is positive semi-definite.

- $\lambda_{1}=0$ is an eigenvalue with multiplicity $r$ of $L \otimes I_{r}$ and it has $r$ different eigenvectors defined by $\mathbf{1}_{n} \otimes e_{i}, i=$ $1, \ldots, r$ where $e_{i} \in \mathbb{R}^{r}$ is the column vector whose $i^{t h}$ components equals 1 and all the others are 0 .

- there exists an orthonormal matrix $T \in \mathbb{R}^{n \times n}$ (i.e. $T T^{\top}=T^{\top} T=I_{n}$ ) such that

$$
T L T^{\top}=D=\operatorname{diag}\left(\lambda_{1}, \lambda_{2}, \ldots, \lambda_{n}\right)
$$

Definition 2: The $n$ singularly perturbed systems defined by (1) achieve asymptotic synchronization using local information if there exists a state feedback controller of the form

$$
\begin{array}{r}
u_{i}(t)=K_{1} \sum_{j=1}^{n} g_{i, j}\left(x_{i}(t)-x_{j}(t)\right)+ \\
K_{2} \sum_{j=1}^{n} g_{i, j}\left(z_{i}(t)-z_{j}(t)\right) \\
K_{1} \in \mathbb{R}^{m \times n_{x}}, K_{2} \in \mathbb{R}^{m \times n_{z}}
\end{array}
$$

such that

$\lim _{t \rightarrow \infty}\left\|x_{i}(t)-x_{j}(t)\right\|=0$ and $\lim _{t \rightarrow \infty}\left\|z_{i}(t)-z_{j}(t)\right\|=0$.

The main goal of this paper is the characterization of the feedback controllers that use local information and asymptotically synchronize the singularly perturbed systems defined by (1). In order to do that we firstly define the collective dynamics describing the behavior of the overall network of $n$ feedback coupled systems.

Let us denote by $x(t) \in \mathbb{R}^{n \cdot n_{x}}$ and $z(t) \in \mathbb{R}^{n \cdot n_{z}}$ the vectors collecting the states $x_{i}(t)$ and $z_{i}(t), i=1, \ldots, n$, respectively (i.e. $x(t)=\left(x_{1}(t)^{\top}, \ldots, x_{n}(t)^{\top}\right)^{\top}$ and $\left.z(t)=\left(z_{1}(t)^{\top}, \ldots, z_{n}(t)^{\top}\right)^{\top}\right)$.

Remark 2: Let us observe that the asymptotic synchronization is equivalent with

$$
\lim _{t \rightarrow \infty}\left(L \otimes I_{n_{x}}\right) x(t)=0, \text { and } \lim _{t \rightarrow \infty}\left(L \otimes I_{n_{z}}\right) z(t)=0
$$

and since $T L=D T$, the synchronization is also characterized by

$$
\begin{aligned}
& \lim _{t \rightarrow \infty}\left(D \otimes I_{n_{x}}\right)\left(T \otimes I_{n_{x}}\right) x(t)=0, \text { and } \\
& \lim _{t \rightarrow \infty}\left(D \otimes I_{n_{z}}\right)\left(T \otimes I_{n_{z}}\right) z(t)=0
\end{aligned}
$$

Replacing (2) in (1) one obtains the following collective closed-loop dynamics: 


$$
\left\{\begin{aligned}
\dot{x}(t) & =\bar{A}_{11} x(t)+\bar{A}_{12} z(t) \\
\epsilon \dot{z}(t) & =\bar{A}_{21} x(t)+\bar{A}_{22} z(t)
\end{aligned}\right.
$$

where

$$
\begin{aligned}
& \bar{A}_{11}=I_{n} \otimes A_{11}-\left(I_{n} \otimes B_{1} K_{1}\right)\left(L \otimes I_{n_{x}}\right), \\
& \bar{A}_{12}=I_{n} \otimes A_{12}-\left(I_{n} \otimes B_{1} K_{2}\right)\left(L \otimes I_{n_{z}}\right) \\
& \bar{A}_{21}=I_{n} \otimes A_{21}-\left(I_{n} \otimes B_{2} K_{1}\right)\left(L \otimes I_{n_{x}}\right), \\
& \bar{A}_{22}=I_{n} \otimes A_{22}-\left(I_{n} \otimes B_{2} K_{2}\right)\left(L \otimes I_{n_{z}}\right)
\end{aligned}
$$

We note that one has no guaranty that the matrix $\bar{A}_{22}$ is invertible. Therefore, the well posedness of the closedloop system(4) has also to be ensured by the choice of the controller gains.

A final change of variable

$$
\tilde{x}(t)=\left(T \otimes I_{n_{x}}\right) x(t), \quad \tilde{z}(t)=\left(T \otimes I_{n_{z}}\right) z(t)
$$

allows at rewriting the collective dynamics (4) as

$$
\left\{\begin{aligned}
\dot{\tilde{x}}(t) & =\tilde{A}_{11} \tilde{x}(t)+\tilde{A}_{12} \tilde{z}(t) \\
\epsilon \dot{\tilde{z}}(t) & =\tilde{A}_{21} \tilde{x}(t)+\tilde{A}_{22} \tilde{z}(t)
\end{aligned}\right.
$$

where

$$
\begin{aligned}
& \tilde{A}_{11}=I_{n} \otimes A_{11}-\left(I_{n} \otimes B_{1} K_{1}\right)\left(D \otimes I_{n_{x}}\right), \\
& \tilde{A}_{12}=I_{n} \otimes A_{12}-\left(I_{n} \otimes B_{1} K_{2}\right)\left(D \otimes I_{n_{z}}\right) \\
& \tilde{A}_{21}=I_{n} \otimes A_{21}-\left(I_{n} \otimes B_{2} K_{1}\right)\left(D \otimes I_{n_{x}}\right), \\
& \tilde{A}_{22}=I_{n} \otimes A_{22}-\left(I_{n} \otimes B_{2} K_{2}\right)\left(D \otimes I_{n_{z}}\right)
\end{aligned}
$$

\section{Discussion}

1) Using the properties of Kronecker product, the closedloop system (5) can be decoupled in $n$ independent singularly perturbed systems. Precisely, one uses that for any matrices $M, N$ of appropriate dimension we have

$$
\begin{gathered}
I_{n} \otimes M-\left(I_{n} \otimes N\right)\left(D \otimes I_{m}\right)=I_{n} \otimes M-D \otimes N \\
\quad=\operatorname{diag}(M, \ldots, M)-\operatorname{diag}\left(\lambda_{1} N, \ldots, \lambda_{n} N\right) \\
=\operatorname{diag}\left(M-\lambda_{1} N, \ldots, M-\lambda_{n} N\right)
\end{gathered}
$$

yielding

$$
\begin{aligned}
& \tilde{A}_{11}=\operatorname{diag}\left(A_{11}-\lambda_{1} B_{1} K_{1}, \ldots, A_{11}-\lambda_{n} B_{1} K_{1}\right), \\
& \tilde{A}_{12}=\operatorname{diag}\left(A_{12}-\lambda_{1} B_{1} K_{2}, \ldots, A_{12}-\lambda_{n} B_{1} K_{2}\right) \\
& \tilde{A}_{21}=\operatorname{diag}\left(A_{21}-\lambda_{1} B_{2} K_{1}, \ldots, A_{21}-\lambda_{n} B_{2} K_{1}\right), \\
& \tilde{A}_{22}=\operatorname{diag}\left(A_{22}-\lambda_{1} B_{2} K_{2}, \ldots, A_{22}-\lambda_{n} B_{2} K_{2}\right)
\end{aligned}
$$

Therefore, the closed-loop system (5) is equivalent with

$$
\left\{\begin{aligned}
\dot{\tilde{x}}_{i}(t) & =\left(A_{11}-\lambda_{i} B_{1} K_{1}\right) \tilde{x}_{i}(t)+\left(A_{12}-\lambda_{i} B_{1} K_{2}\right) \tilde{z}_{i}(t) \\
\epsilon \dot{\tilde{z}}_{i}(t) & =\left(A_{21}-\lambda_{i} B_{2} K_{1}\right) \tilde{x}_{i}(t)+\left(A_{22}-\lambda_{i} B_{2} K_{2}\right) \tilde{z}_{i}(t) \\
i & =1, \ldots, n
\end{aligned}\right.
$$

2) The asymptotic synchronization problem with local information becomes a problem of simultaneous stabilization of systems in (6) for $i=2, \ldots, n$. Indeed (3) can be seen as

$\lim _{t \rightarrow \infty}\left(D \otimes I_{n_{x}}\right) \tilde{x}(t)=0$ and $\lim _{t \rightarrow \infty}\left(D \otimes I_{n_{z}}\right) \tilde{z}(t)=0$

but since $D=\operatorname{diag}\left(\lambda_{1}, \ldots, \lambda_{n}\right)$ with $\lambda_{1}=0$ the condition is transformed as

$$
\lim _{t \rightarrow \infty} \tilde{x}_{i}(t)=0 \text { and } \lim _{t \rightarrow \infty} \tilde{z}_{i}(t)=0, i=2, \ldots n
$$

3) We emphasize that from the definition of $T$, the following also hold $x(t)=\left(T^{\top} \otimes I_{n_{x}}\right) \tilde{x}(t)$ and $z(t)=$ $\left(T^{\top} \otimes I_{n_{z}}\right) \tilde{z}(t)$. Thus, the synchronization manifold depends on the dynamics of $(\tilde{x}(t), \tilde{z}(t))$. Precisely, if the system

$$
\left\{\begin{aligned}
\dot{\tilde{x}}_{1}(t) & =A_{11} \tilde{x}_{1}(t)+A_{12} \tilde{z}_{1}(t) \\
\epsilon \dot{\tilde{z}}_{1}(t) & =A_{21} \tilde{x}_{1}(t)+A_{22} \tilde{z}_{1}(t) \\
i & =1, \ldots, n
\end{aligned}\right.
$$

has a stable equilibrium point $\left(\tilde{x}^{*}, \tilde{z}^{*}\right)$, then systems (1) will asymptotically reach a finite consensus. If (7) is unstable then all the systems in (1) will synchronize on divergent trajectories.

4) The well posedness of system (4) is equivalent with the one of system (5) which in turn is ensured if all systems in (6) are well posed. Let us notice that for $i=1$ the system is well posed due to Assumption 1 . The rest of the systems in (5) are well posed if $K_{2}$ is chosen such that $A_{22}-\lambda_{i} B_{2} K_{2}$ is invertible for $i=2, \ldots, n$.

\section{CONTROL DESIGN}

Before giving our main result let us introduce some notation that allows at completely decouple the slow and fast dynamics that occur in the overall system. This is done by following the classical singular perturbation design (see [2] for instance). Let us consider the slow systems

$$
\left\{\begin{array}{c}
\dot{\tilde{x}}_{i, s}(t)=\left(A_{0}-\lambda_{i} B_{0} K_{0}\right) \tilde{x}_{i, s}(t), \quad \tilde{x}_{i, s}(0)=\tilde{x}_{i}(0) \\
\tilde{z}_{i, s}(t)=-A_{22}^{-1}\left(A_{21}-\lambda_{i} B_{2} K_{0}\right) \tilde{x}_{i, s}(t) \\
i=1, \ldots, n
\end{array}\right.
$$

where

$$
A_{0}=A_{11}-A_{12} A_{22}^{-1} A_{21}, \quad B_{0}=B_{1}-A_{12} A_{22}^{-1} B_{2} .
$$

Consequently, the corresponding fast systems are

$\epsilon \dot{\tilde{z}}_{i, f}(t)=\left(A_{22}-\lambda_{i} B_{2} K_{2}\right) \tilde{z}_{i, f}(t), \quad \tilde{z}_{i, f}(0)=\tilde{z}_{i}(0)-\tilde{z}_{i, s}(0)$

Using the notation above the following result holds.

Theorem 3: Let $K_{2}$ and $K_{0}$ be designed such that for $i=2, \ldots, n$ the matrices $A_{22}-\lambda_{i} B_{2} K_{2}$ and $A_{0}-\lambda_{i} B_{0} K_{0}$ are all Hurwitz. Then, there exists $\epsilon^{*}>0$ such that the controllers (2) with 


$$
K_{1}=\left(I_{m}-K_{2} A_{22}^{-1} B_{2}\right) K_{0}+K_{2} A_{22}^{-1} A_{21} .
$$

asymptotically synchronize with local information the systems (1).

Proof: Following [2], the choice of $K_{1}$ and $K_{2}$ as in the statement above ensures that for $i=2, \ldots, n$ the systems in (6) are asymptotically stable. Moreover, for all $\epsilon \in\left(0, \epsilon^{*}\right]$ and all $t \geq 0$ one has

$$
\begin{aligned}
& \tilde{x}_{i}(t)=\tilde{x}_{i, s}(t)+O(\epsilon), \\
& \tilde{z}_{i}(t)=-A_{22}^{-1}\left(A_{21}-\lambda_{i} B_{2} K_{0}\right) \tilde{x}_{i, s}(t)+\tilde{z}_{i, f}(t)+O(\epsilon) .
\end{aligned}
$$

We recall here that the asymptotic synchronization is equivalent with

$$
\lim _{t \rightarrow \infty}\left(L \otimes I_{n_{x}}\right) x(t)=0 \text { and } \lim _{t \rightarrow \infty}\left(L \otimes I_{n_{z}}\right) z(t)=0
$$

which hold true since

$$
\left(L \otimes I_{n_{x}}\right) x(t)=\left(D \otimes I_{n_{x}}\right) \tilde{x}(t)=\left[\begin{array}{c}
0 \\
\lambda_{2} \tilde{x}_{2} \\
\vdots \\
\lambda_{n} \tilde{x}_{n}
\end{array}\right]
$$

and

$$
\left(L \otimes I_{n_{z}}\right) z(t)=\left(D \otimes I_{n_{z}}\right) \tilde{z}(t)=\left[\begin{array}{c}
0 \\
\lambda_{2} \tilde{z}_{2} \\
\vdots \\
\lambda_{n} \tilde{z}_{n}
\end{array}\right]
$$

Remark 3: Theorem 3 basically says that in order to asymptotically synchronize systems in (1) we have to separately synchronize the fast and slow dynamics by stabilizing the dynamics of the error between the different systems.

Corollary 1: Let $K_{0}$ be designed such that for $i=2, \ldots, n$ the matrices $A_{0}-\lambda_{i} B_{0} K_{0}$ are Hurwitz. If the matrix $A_{22}$ is Hurwitz the controllers $u_{i}$ in (2) with $K_{1}=K_{0}$ and $K_{2}=\mathbf{0}_{m \times n_{z}}$, asymptotically synchronize the systems (1).

Remark 4: We note that $A_{22}$ is Hurwitz corresponds to the case in which the fast dynamics is stable. In this case, the control design can be directly realized by approximating the dynamics (1) with the corresponding decoupled slow and fast dynamics. Thus, we have to synchronize only the slow linear dynamics representing the reduced systems in (1).

We also note that, when $A_{22}$ is Hurwitz the dynamics (7) can be approximated by its decoupled version in fast and slow dynamics.

Using Theorem 3 we reduce the synchronization problem to the one of state feedback simultaneous stabilization (SFSS) of linear systems [20], [21]. Simultaneous stabilization is in general a difficult undecidable problem that received a lot of attention [22], [23]. Nevertheless, we are facing a very particular SFSS problem that can be formulated as follows.

Problem 1: For $A \in \mathbb{R}^{p \times p}, B \in \mathbb{R}^{p \times m}$ and $0<\lambda_{2} \leq \lambda_{3} \leq \ldots \leq \lambda_{n}$ find $K \in \mathbb{R}^{m \times p}$ such that for $i=2, \ldots, n$ the matrices $A-\lambda_{i} B K$ are Hurwitz.

It is obvious that the problem is feasible as far as the states $x_{i}(t)$ and $z_{i}(t)$ are scalar since depending on the sign of $B$ we have to render negative only the scalar $A-\lambda_{2} B K$ or $A-\lambda_{n} B K$. When $x_{i}(t)$ and $z_{i}(t)$ are not scalars we have to simultaneously render the $n-1$ matrices $A-\lambda_{i} B K$ Hurwitz.

Proposition 4: If the pair $(A, B)$ is controllable then there exists $K$ solving Problem 1.

Before giving the proof of this result let us state the following.

Lemma 5: The matrix $M \in \mathbb{R}^{n \times n}$ is Hurwitz if $\forall x \in$ $\mathbb{R}^{n} \backslash\left\{\mathbf{0}_{n}\right\}$ one has $x^{\top} M x<0$.

Proof: Let $\nu$ be an eigenvalue of $M$. It means (see [24]) that there exists $x \in \mathbb{C}^{n} \backslash\left\{\mathbf{0}_{n}\right\}$ such that $\nu=\bar{x}^{\top} M x$ where $\bar{x}$ stands for the complex conjugate of $x$. Let us consider $r, q \in \mathbb{R}^{n}$ the real and imaginary part of $x \in \mathbb{C}^{n}$. Since $M$ is a matrix with real components, the following computation is straightforward

$$
2 \Re(\nu)=\nu+\bar{\nu}=\bar{x}^{\top} M x+x^{\top} M \bar{x}=2\left(r^{\top} M r+q^{\top} M q\right)
$$

which leads to $\Re(\nu)<0$ since $r, q \in \mathbb{R}^{n}$ and at least one of them is not $\mathbf{0}_{n}$.

Proof of Proposition 4: In order to prove the existence of $K$ solving Problem 1 we show that there exists $K$ such that $\forall x \in \mathbb{R}^{p} \backslash\left\{\mathbf{0}_{p}\right\}, \forall i=2, \ldots, n$ one has

$$
x^{\top}\left(A+\lambda_{i} B K\right) x<0 .
$$

If $(A, B)$ is controllable then $\left(A, \lambda_{2} B\right)$ is controllable too. Let us notice that $0<\lambda_{2} \leq \lambda_{3} \leq \ldots \leq \lambda_{n}$. Therefore, the following makes sense for $i=2, \ldots, n$

$$
A+\lambda_{i} B K=\frac{\lambda_{i}}{\lambda_{2}}\left(A+\lambda_{2} B K+\left(\frac{\lambda_{2}}{\lambda_{i}}-1\right) A\right) .
$$

Considering

$$
\mu=\max _{i=2, \ldots, n}\left\|\left(\frac{\lambda_{2}}{\lambda_{i}}-1\right) A\right\|
$$

one has

$$
x^{\top}\left(\frac{\lambda_{2}}{\lambda_{i}}-1\right) A x \leq \mu\|x\|=x^{\top} \mu I_{p} x
$$

Therefore,

$$
x^{\top}\left(A+\lambda_{i} B K\right) x \leq \frac{\lambda_{i}}{\lambda_{2}} x^{\top}\left(A+\lambda_{2} B K+\mu I_{p}\right) x
$$

Since $\left(A, \lambda_{2} B\right)$ is controllable we can choose $K$ such that all the eigenvalues $\eta_{j}, j=1, \ldots, p$ of $A+\lambda_{2} B K$ are real, different one from another and smaller than $-\mu$. Doing so, there exists a nonsingular matrix $T \in \mathbb{R}^{p}$ such that

$$
T^{-1}\left(A+\lambda_{2} B K\right) T=\operatorname{diag}\left(\eta_{1}, \ldots, \eta_{p}\right)
$$


meaning that

$T^{-1}\left(A+\lambda_{2} B K+\mu I_{p}\right) T=\operatorname{diag}\left(\eta_{1}+\mu, \ldots \eta_{p}+\mu\right)<0$.

Using (10) and the previous inequality we conclude that (8) holds.

Remark 5: It is noteworthy that the controller design can be done in a decentralized manner since each agent needs to compute only norm of $A_{22}$ and $A_{0}$ in order to get $K_{2}$ and $K_{0}$ respectively.

Another practical way of finding the gain $K$ is by solving the following $n-1$ linear matrix inequalities (LMIs):

$$
\left(A-\lambda_{i} B K\right)^{\top} P+P\left(A-\lambda_{i} B K\right)<0, i=2, \ldots, n
$$

where $P=P^{\top}>0$ is a matrix to be found if it exists. More exactly, one has $P=S^{-1}$ and $K=R S^{-1}$ if $S=$ $S^{\top}>0, R \in \mathbb{R}^{m \times p}$ solve the following standard LMIs [25]:

$$
S A^{\top}+A S-\lambda_{i}\left(R^{\top} B^{\top}+B R\right)<0, i=2, \ldots, n
$$

Note that the previous LMIs are used to numerically find the value of $K$ that simultaneously renders $A-\lambda_{i} B K$ Hurwitz for $i=2, \ldots, n$.

\section{ILLUSTRATIVE EXAMPLES}

In this section, we consider the synchronisation of three agents described by an undirected graph $\mathcal{G}$. One representative example is given for illustration.

The system is given by (1) where :

$$
\begin{gathered}
A_{11}=\left(\begin{array}{cc}
2,5 & -6 \\
-2 & 2
\end{array}\right), A_{12}=\left(\begin{array}{cc}
2 & 3 \\
0 & -2
\end{array}\right) \\
A_{21}=\left(\begin{array}{ll}
0,5 & 2 \\
-1 & 1
\end{array}\right), A_{22}=\left(\begin{array}{cc}
-2 & 1 \\
0 & -1
\end{array}\right) \\
B_{1}=\left(\begin{array}{l}
2 \\
1
\end{array}\right), B_{2}=\left(\begin{array}{l}
1 \\
1
\end{array}\right)
\end{gathered}
$$

To each agent we assign a vector state having 4 components characterized by slow and fast dynamics. For any agent $i \in$ $\{1, \ldots, n\}$, let us denote by $\left[x_{i, 1}, x_{i, 2}\right]^{\top}$ and $\left[z_{i, 1}, z_{i, 2}\right]^{\top}$ its slow and fast state' components, respectively. The Laplacian matrix describing the undirected topology of the graph $\mathcal{G}$ is defined by:

$$
L=\left(\begin{array}{ccc}
2 & -1 & -1 \\
-1 & 2 & -1 \\
-1 & -1 & 2
\end{array}\right)
$$

In simulation we fix $\epsilon=0.001$ and all the components of the initial condition are chosen within $[-4,5]$. Since $A_{22}$ is Hurwitz we have to solve numerically 2 LMIs to stabilize the dynamics (6) such that $K_{1}=K_{0}$. Figure 1 highlights the synchronization of the slow dynamics for an $\epsilon$ sufficiently small.

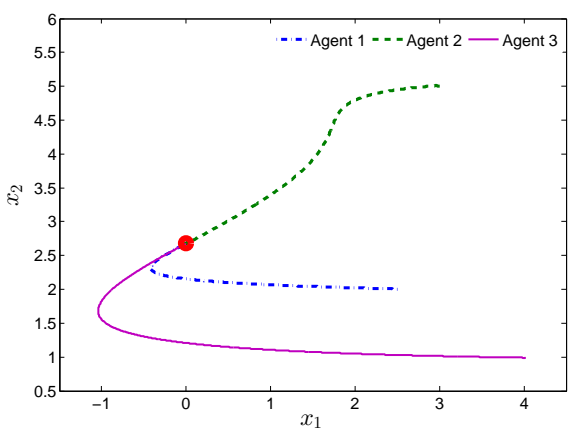

Fig. 1. Trajectories of the $x$ components of system (4). The red bullet is the stable equilibrium $x^{*}$ of (7).

To highlight the fast transient dynamics, we represent the evolution of the fast states function of the slow ones.
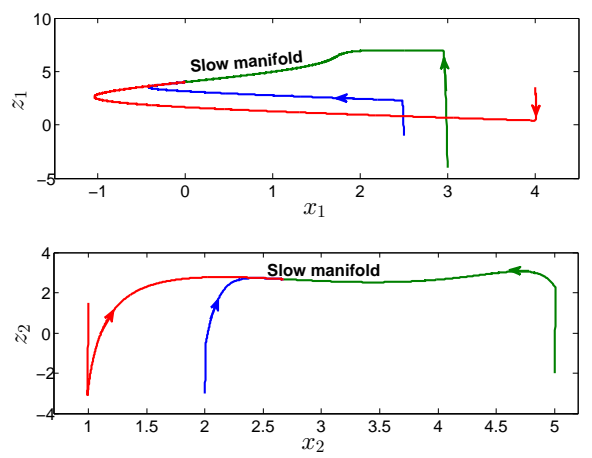

Fig. 2. Slow and fast manifolds

Figures 3 and 4 show that the slow and fast models approximate the decoupled system within $O(\epsilon)$ neighborhood.

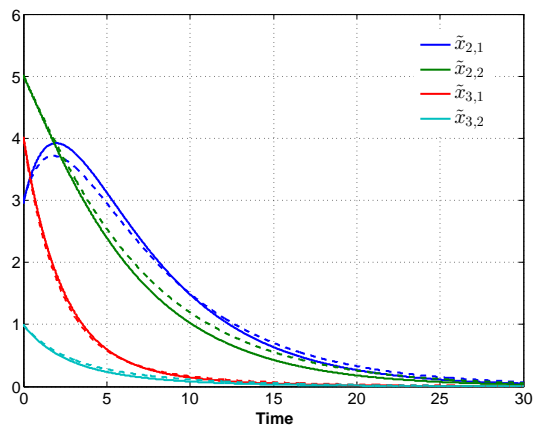

Fig. 3. The trajectories of $\tilde{x}$ (solid) and $\tilde{x}_{s}$ (dotted)

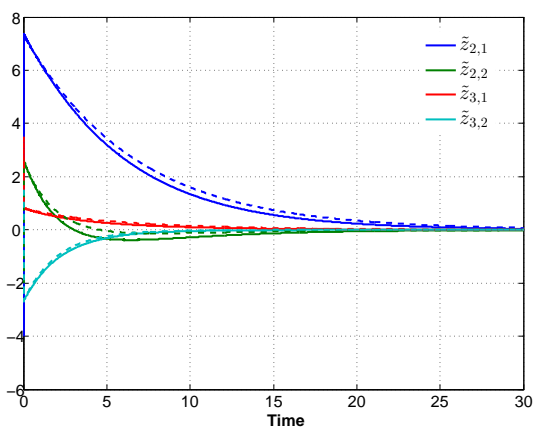

Fig. 4. The trajectories of $\tilde{z}$ (solid) and $\tilde{z}_{s}+\tilde{z}_{f}$ (dotted) 
To emphasize the fast dynamics we also plot in Figure 7 the fast part of $z$ variables.

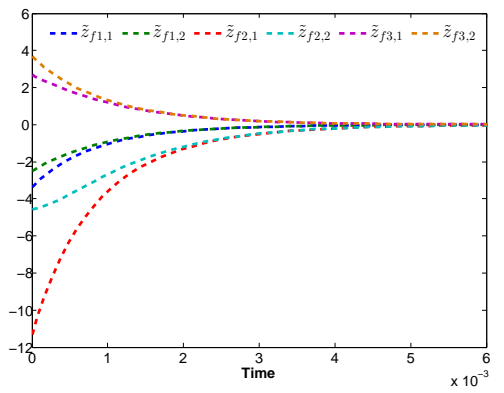

Fig. 5. The fast part of $\tilde{z}$ components $\tilde{z}_{f_{i}} \forall i \in\{1, \ldots, n\}$

The synchronization of the two-time scale model (1) ensures the stabilization of the errors between the slow states as shown in figure 6.

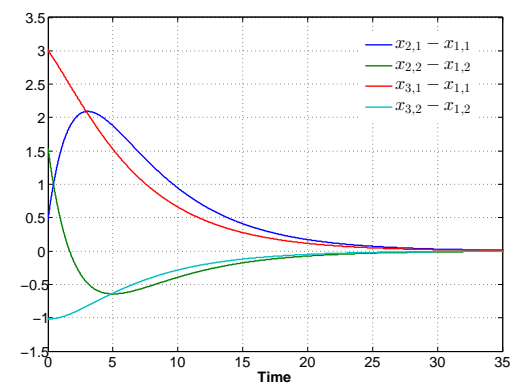

Fig. 6. The differences $x_{i}-x_{j}, \forall i \neq j \in\{1, \ldots, n\}$

The trajectories of system are also provided in figure 7
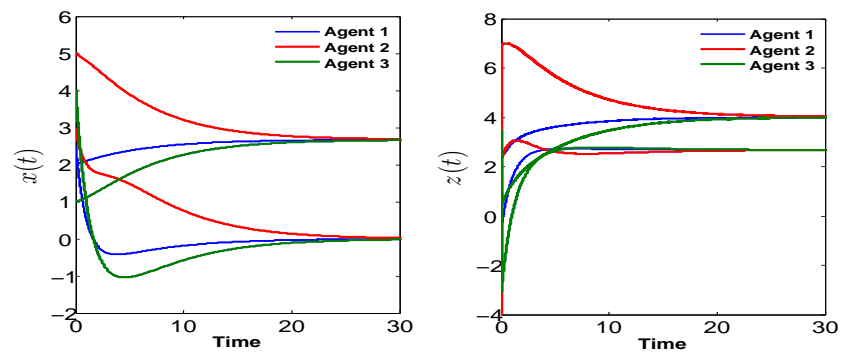

Fig. 7. The trajectories of system

\section{CONCLUSIONS}

This paper provides a decentralized controller design for the synchronization of coupled singularly perturbed systems. The feedback controller use only local information. The standard singular perturbation analysis and design are adapted to synchronization purposes. The controller design is computationally oriented since it is obtained by solving some LMIs. We also show that under mild assumptions the LMIs are always feasible. One numerical example illustrates the method implementation. Stabilization of linear and nonlinear singularly perturbed systems for directed graphs and timevarying topologies are is our future focus.

\section{REFERENCES}

[1] D. Naidu, "Singular perturbations and time scales in control theory and applications: an overview," Dynamics of Continuous, Discrete \& Impulsive Systems. Series B. Applications \& Algorithms, vol. 9, no. 2 , pp. 233-278, 2002.

[2] P. Kokotović, H. Khalil, and J. O'Reilly, Singular perturbation methods in control: analysis and design. SIAM Series in Classics and Applied Mathematics, 1999.

[3] H. Khalil, Nonlinear Systems (Third Edition). Prentice Hall, 2001.

[4] L.Chen and K. Aihara, "A model of periodic oscillation for genetic regulatory systems," IEEE Transactions on Circuits and Systems, vol. 49, no. 10, pp. 1429-1436, 2002.

[5] A. Hodgkin and A. Huxley, "A quantitative description of membrane current and its application to conduction and excitation in nerve," The Journal of Physiology, vol. 117, pp. 500-544, 1952.

[6] I. Malloci, "Two time scale switched systems: Application to steering control in hot strip mills," Ph.D. dissertation, Univ. Lorraine - CRAN UMR 7039, 2009.

[7] G. Garcia, J. Daafouz, and J. Bernussou, "The infinite time near optimal decentralized regulator problem for singularly perturbed systems: a convex optimization approach." Automatica, vol. 38, no. 8, pp. 1397 1406, 2002

[8] M.Alwan, X. Liu, and B. Ingalls, "Exponential stability of singularly perturbed switched systems with time delay," Nonlinear Analysis: Hybrid Systems, vol. 2, no. 3, pp. 913-921, 2008.

[9] I. Malloci, J. Daafouz, and C. Iung, "Stabilization of continuous-time singularly perturbed switched systems," in Proc. of the 48th IEEE Conference on Decision and Control, 2009.

[10] G. Hek, "Geometric singular perturbation theory in biological practice," Journal of Mathematical Biology, vol. 60, pp. 347-386, 2010.

[11] Y. Kuramoto, Chemical Oscillations, Waves and Turbulence. Springer, New York, 1984.

[12] L. M. Pecora and T. L. Carroll, "Master stability functions for synchronized coupled systems," Phys. Rev. Lett., vol. 80, no. 10, pp. 2109-2112, 1998

[13] S. H. Strogatz, Sync: The Emerging Science of Spontaneous Order. Hyperion Press, 2003.

[14] W. Michiels, I.-C. Morărescu, and S.-I. Niculescu, "Consensus problems with distributed delays, with application to traffic flow models," SIAM Journal on Control and Optimization, vol. 48, no. 1, pp. 77-101, 2009

[15] I.-C. Morărescu, W. Michiels, and M. Jungers, "Synchronization of coupled nonlinearoscillators with shifted gamma-distributed delays," in Proceedings of American Control Conference, 2013.

[16] J. Chow and P. Kokotović, "Time scale modeling of sparse dynamic networks," IEEE Transactions on Automatic Control, vol. 30, no. 8 , pp. 714-722, 1985.

[17] E. Biyık and M. Arcak, "Area aggregation and time-scale modeling for sparse nonlinear networks," Systems \& Control Letters, vol. 57, no. 2, pp. 142-149, 2007.

[18] D. Romeres, f. Dörfler, and F. Bullo, "Novel results on slow coherency in consensus and power networks," in Proc. of the European Control Conference (ECC), 2013.

[19] C. Godsil and G. Royle, Algebraic Graph Theory. Springer-Verlag, New-York, 2001

[20] R. Luke, P. Dorato, and C. Abdallah, "A survey of state feedback simultaneous stabilization techniques," in Proceedings of 2nd World Automation Congress, 1996.

[21] F. Saadatjoo, V. Derhami, and S. Karbassi, "Simultaneous control of linear systems by state feedback," Computers \& Mathematics with Applications, vol. 58, no. 1, pp. 154-160, 2009.

[22] V. Blondel, Simultaneous stabilization of linear systems, ser. Lecture Notes in Control and Information Sciences. Springer-Verlag, 1994, vol. 191.

[23] D. Henrion, S. Tarbouriech, and M. Sebek, "Rank-one lmi approach to simultaneous stabilization of linear systems," Systems \& Control Letters, vol. 38, no. 2, pp. 79-89, 1999.

[24] H. Wielandt, "On the eigenvalues of $\mathrm{a}+\mathrm{b}$ and ab," Journal of research of the National Bureau of Standards - B. Mathematical Sciences, vol. 778, no. 1 \& 2, 1973.

[25] S. Boyd, L. El Ghaoui, E. Feron, and V. Balakrishnan, Linear Matrix Inequalities in system and control theory. SIAM, 1994 